\title{
Microbiological monitoring of continuous positive airway pressure and resuscitation equipment in very-low birth weight infants
}

\author{
Torben Christian Winking ${ }^{1}$, Jörg Wüllenweber ${ }^{2}$, Frank Kipp ${ }^{3}$ and Esther Rieger-Fackeldey ${ }^{1}$
}

BACKGROUND: To investigate the colonization of respiratory equipment and the rate of respiratory infections of very-low birth weight (VLBW) infants.

METHODS: The prospective study includes 26 VLBW infants on continuous airway pressure (CPAP) from September until December 2012. Swabs from respiratory equipment and colonization/infections were evaluated.

RESULTS: A total of 603 swabs was cultured with 298 isolates; $59 \%$ of cultures from CPAP equipment $(n=337 ; 95 \%$ confidence interval $(\mathrm{Cl} ; 54 ; 64))$ and $19 \%$ from ambu bags $(n=51 ; 95 \% \mathrm{Cl}(14 ; 24))$ were positive. Overall, 181/201 CPAP prongs and masks hosted 221 microorganisms. Colonization on days 3 and 7 were $93 \%$ and $87 \%$, respectively, with an increase in pathogens and a decrease in skin flora (79\% vs. 68\%). Comparing the 58 paired swab results from days 3 and 7 showed an increase in Gram-negative bacteria $(P=0.014)$. Eighteen infants had positive weekly screening results, with similar colonization of CPAP equipment, dominated by Enterobacteriacae. Pneumonia was diagnosed in two infants. CONCLUSION: Of the CPAP equipment close to the patient, $90 \%$ was colonized with microorganisms increasing during 1 week of CPAP. The pathogens were dominated by gastrointestinal bacteria, and persisted over weeks. Frequent cleaning did not prevent pneumonia, although pneumonia rates were rare.

V ery-low birth weight (VLBW) and, especially, extremely low birth weight infants are a very vulnerable group of patients and are prone to infections (incidence 20-30\%) (1). Sepsis is the most common type of nosocomial infections in preterm infants, (2) and ventilator-associated pneumonia is a serious complication in mechanically ventilated infants (3).

Colonization of the skin is a well-known risk factor for nosocomial infections in preterm infants. Often, the medical equipment used for a preterm infant is colonized with the same microorganisms as the skin (4). As early as 2006, Graham et al. reported that nasal cannulae are a risk factor for Gram-negative sepsis (5), although even earlier in 1991 Gupta et al. reported that the colonization of the resuscitation equipment is a risk factor for hospital-acquired infection (6). Meinich Petersen et al. reported that nasogastric feeding tubes yield high concentrations of facultative pathogenic bacteria even 1 day after insertion (7).

The incidence of pneumonia in VLBW infants is 0.9 per 1,000 patient days (2) and is ventilator-associated in $81 \%$ of all cases. Microaspiration of oropharyngeal secretions is the most common reason (8). Non-invasive ventilation and CPAP have a lower risk for pneumonia than invasive ventilation (1.0 vs. 2.7 per 1,000 patient days); however, Aly et al. (4) reported that the nasal flora changes under CPAP. The physiological nasal flora mainly consists of coagulasenegative staphylococci (CoNS); however, with continuous positive pressure ventilation (CPAP), Gram-negative bacteria can be detected in $38 \%$ of the cases. Nevertheless, there are few data concerning the detailed colonization of respiratory equipment over weeks and resulting infections.

We analyzed the time until and the duration of colonization of different parts of the CPAP equipment and the ambu bags of preterm infants, type of isolates, conformity with weekly screening swab results from the pharynx and anus, and respiratory infections with colonizing isolates.

\section{Methods}

A prospective observational study was conducted in the neonatal division of the University Children's Hospital of Muenster between 1 September and 31 December 2012. All preterm infants with a birth weight $<1,500 \mathrm{~g}$ (VLBW infants), inborn and outborn, were included in the study. We excluded infants with a stay of fewer than 3 days in the unit. Swabs from CPAP and resuscitation equipment of these infants were obtained from the intensive care unit and the intermediate care unit. Swabs from the equipment were taken during nursing rounds by the attending nurse. As with routine bacteriological monitoring once per week, this investigation was part of the quality control in the unit, and therefore no ethical approval and no informed consent were obtained.

The swabs were obtained from the inside of prongs/masks, connectors, inspiratory limbs $(20 \mathrm{~cm}$ distance to water pot), and water pots (entry of inspiratory limb into the pot) of patients' respiratory equipment during their time on CPAP. The swabs from ambu bags were obtained from masks and connectors as long as the patient was on CPAP. We used the Transwab system (MW \& E,

${ }^{1}$ Department of General Pediatrics, University Children's Hospital Munster, Munster, Germany; ${ }^{2}$ Institute of Medical Microbiology, University Hospital Munster, Munster, Germany; ${ }^{3}$ Institute for Hygiene, University Hospital Munster, Munster, Germany. Correspondence: Esther Rieger-Fackeldey (esther.riegerfackeldey@tum.de) 


\section{Microbiol monitoring in infants on CPAP Articles}

Corsham, England) for all pieces of equipment except for prongs, where we used the thinner Mastaswab system (Mast Diagnostica $\mathrm{GmbH}$, Reinfeld, Germany). The swabs were moistured with $\mathrm{NaCl}$ $0.9 \%$ before application when the surface of the device was dry. The swabs from prongs and masks were obtained on days 3 and 7, from connectors on days 7 and 14, and from inspiratory limbs and water pots on day 14 . The masks and prongs were cleaned with $\mathrm{NaCl} 0.9 \%$ about four times per day during nursing rounds and the swabs were taken before cleaning. The prongs were changed after 7 days and the rest of the equipment after 14 days. The number of the swabs obtained varied per patient according to his/her time on CPAP.

Routine swabs from the pharynx and anus were obtained every Monday from the intensive care unit as recommended by the national guidelines from 2013 on (9) we had already implemented from 2012 on. Patients on CPAP who were transferred to the intermediate care unit did not receive the routine swabs any more, but continued with the swabs obtained from the CPAP equipment. Infants with respiratory deterioration were intubated and ventilated and tracheal aspirates were taken immediately after intubation and every Monday as long as they were intubated.

All swabs and aspirates were transferred to the Institute of Medical Microbiology of the University of Muenster at room temperature on the same day. Specimens were cultured on Columbia-, MacConkey-, and Blood-Agar media. In addition, a culture in dextrose bouillon was started. Tracheal aspirates were inoculated with a loop on the agar plates. Agar cultures were incubated at $37^{\circ} \mathrm{C}$ for at least $24 \mathrm{~h}$ and at a maximum of $48 \mathrm{~h}$ in the case of no bacterial growth. Cultures on Cimmig- and CHROMagar (BD Diagnostics, Heidelberg, Germany) were used for fungal growth.

Identification of microorganism was by means of VITEK2 (Biomérieux, Marcy-l'Étoile, France) and MALDI-TOF (Matrixassisted laser desorption/ionization-time of flight). Antibiotic sensitivity tests were performed with VITEK2.

Colonization of the CPAP equipment was compared with colonization of the pharynx and anus obtained from weekly screening swabs. The pathogens from tracheal aspirates were

Table 1. Patient demographics.

\begin{tabular}{lc}
\hline Patients, $n$ & 26 \\
Gestational age & \\
Median (min-max) & $26+2(23+6 ; 32+5)$ \\
Birth weight, $g$ & \\
$\quad$ Median (min-max) & $850(495 ; 1,480)$ \\
Gender, $n$ (\%) & \\
Male & $9(35)$ \\
Female & $17(65)$ \\
PDA, $n$ (\%) & $18(69)$ \\
NEC, $n$ (\%) & $1(4)$ \\
BPD, $n$ (\%) & $5(19)$ \\
IVH, $n$ (\%) & $7(27)$ \\
Grade 2 & $2(8)$ \\
Grade 3 & $3(12)$ \\
ROP, $n$ (\%) & $13(50)$ \\
Grade 3 & $4(15)$ \\
Pneumonia, $n$ (\%) & $2(8)$ \\
Study weeks per patient & \\
Median (min-max) & $7(1-19)$ \\
\hline
\end{tabular}

BPD, bronchopulmonary dysplasia; IVH, intraventricular hemorrhage; Max, maximum; Min, minimum; NEC, necrotizing enterocolitis; PDA, persistent ductus arteriosus; ROP, retinopathy of prematurity. compared with results from screening swabs and CPAP equipment swabs.

Sepsis, pneumonia, and necrotizing enterocolitis were diagnosed according to the criteria of the German infections's surveillance system Neo-Kiss (www.nrz-hygiene.de) requiring a combination of clinical, laboratory, and in case of pneumonia and necrotizing enterocolitis, radiological findings. In case of pneumonia, purulent tracheal secretions or the isolation of a respiratory pathogen are optional, but is not an obligatory criterion. This corresponds to CDC criteria that permit the diagnosis of "clinically defined pneumonia" based on clinically and radiologic findings (3).

Antibiotic therapy according to the guidelines of the unit was performed only in case of diagnosed infection. Microbiological findings of the routine screening, but not of the microbiological investigation of the respiratory equipment, were used at the beginning of the antibiotic treatment. The swab collection was continued under antibiotic therapy. Probiotic therapy was not routinely administered.

Statistical evaluation was performed with Sigmaplot 12.0 (Systat Software, San Jose, CA). Data were expressed as the median (range) or counts (percentages). Confidence intervals (CIs) were calculated for larger numbers. The paired results were analyzed by Wilcoxon tests. Risk factors for colonization were determined by $\chi^{2}$-tests. $P<0.05$ was regarded as significant. If an infant had multiple samples, the colonization of all samples of this infant was taken together and this single result of the infant was considered in the analysis. Thus, only one result per infant was considered in the final analysis. For the paired swab test, 58 swab results from days 3 and 7 from the same prongs $(2 \times 58=116)$ could be considered; in all other cases the value of day 7 is missing, because the prong was changed earlier (secretions or blood in the prong).

\section{RESULTS}

A total of 26 VLBW infants were admitted to the neonatal intensive care unit (NICU) during the study period, 21 of them being extremely low birth weight infants. The characteristics and morbidity of the infants are shown in Table 1. Infants were on CPAP for 7.5 (1-19) weeks.

A total of 603 swabs were cultured, of which 337 swabs were from CPAP equipment and 266 swabs were from ambu bags. On 251 swabs 298 isolates were cultured. From CPAP equipment $59 \%$ of the cultures were positive $(n=337 ; 95 \%$ confidence intervals (CI; 54; 64)) and from ambu bags $19 \%$ of the cultures were positive $(n=51 ; 95 \%$ CI $(14 ; 24))$.

Of the 201 CPAP prongs/masks, only 20 were sterile $(10 \%$; 95\% CI 6;14). The remaining 181 prongs/masks hosted 221 microorganisms. Colonization on days 3 and 7 were $93 \%$ vs. $87 \%, n=101$ vs. $n=80)$. On day 3, colonization with normal skin flora was $79 \%(n=86)$ and on day 7 it was $68 \%(n=63)$. Colonization of the CPAP prongs/masks with all organisms is shown in Figure 1 (multiple organisms per device possible). When comparing the 58 paired swab results taken on days 3 and 7 of the prongs/masks, we found an increase in the amount of microorganisms from day 3 to day $7(P=0.021)$ mainly through an increase in the amount of Gram-negative bacteria $(P=0.014)$. One-third of the 45 CPAP connectors was colonized, colonization being higher on day 14 vs. day 7 . After 7 days, three connectors were colonized with skin flora and one with Staphylococcus aureus. After 14 days two connectors were colonized with Gram-negative bacteria and a Nonfermenter $(3 \times$ Enterobacter cloacae and E. vulneris, $1 \times$ 


\section{Articles | Winking et al.}

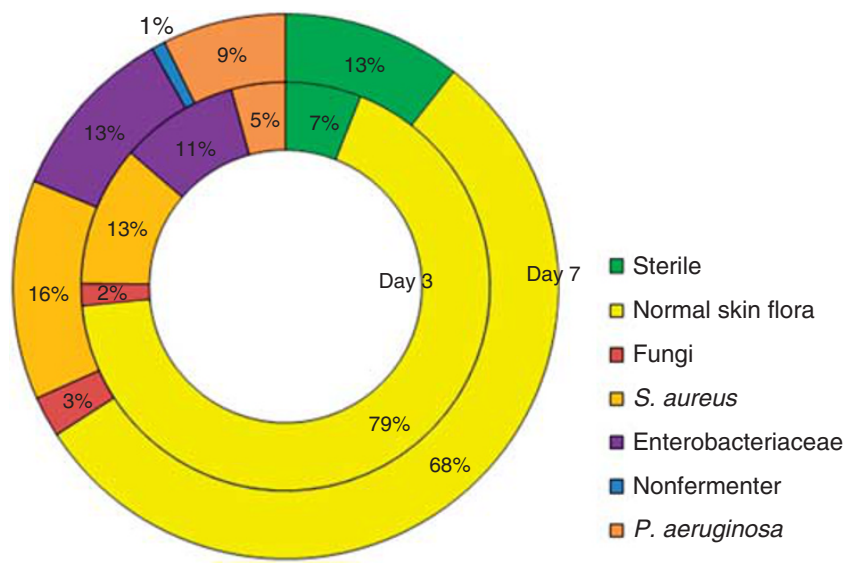

Figure 1. Continuous airway pressure (CPAP) prongs/masks covered with pathogens (\%). Multiple colonization per device was possible. Therefore, the sum of microorganisms is higher than $100 \%$.

Pseudomonas oryzihabitans) and nine connectors with skin flora. Cultures from 45 waterpots and from the interior of 46 inspiratory limbs of 22 patients showed skin flora in four cultures; all others were sterile.

Table 2 shows the type of pathogenic microorganisms colonizing the prongs/masks and connectors. Prongs and masks hosted less skin flora on day 7 than those on day 3 . We found early colonization with Enterobacteriaceae and $P$. aeruginosa.

From 266 swabs from ambu bags, 13 connectors were colonized. Of the swabs from masks, 38 masks were colonized (37\%). On the masks 42 microorganisms were found, most of them being skin flora and environmental flora. On one mask Moraxella osloensis was found, on one S. aureus, and on three Enterobacteriacae were found. The connectors were colonized with skin flora and in one case with E. cloacae.

Eight infants had negative weekly screening results from the pharynx and anus (Table 2); in seven of them no microorganisms were detectable on the CPAP equipment, but one had $S$. aureus on the CPAP equipment. Patients with negative screening results were less ill than the patients with positive screening results; they got their equipment removed, on average, 6 weeks earlier. In 18 infants weekly screening results showed colonization of the pharynx and anus in 87 swabs (Table 3). Infants colonized with Gram-negative bacteria had lower birth weight $(P=0.045)$, lower gestational age $(P=0.045)$, and a longer time of hospital stay $(P=0.032)$. In 11 of the 18 patients, there was colonization of the CPAP equipment, which was equal to colonization of the pharynx/ anus. Seven infants had positive screening results and no colonization of the CPAP equipment (Table 2).

In two patients (8\%) pneumonia was diagnosed during their time on CPAP. In both cases Enterobacteriaceae were isolated in tracheal aspirates after intubation because of respiratory deterioration. In both, the identified E. cloacae also grew at the pharynx/anus and, in one case, it was isolated on the CPAP equipment.
Table 2. Correlation between screening swab and CPAP colonization with facultative pathogenic microorganisms

\begin{tabular}{|c|c|c|}
\hline & $\begin{array}{l}\text { Patients with } \\
\text { negative } \\
\text { screening results } \\
n=8(31 \%)\end{array}$ & $\begin{array}{l}\text { Patients with positive } \\
\text { screening results } \\
n=18(69 \%)\end{array}$ \\
\hline \multicolumn{3}{|l|}{ Gestational age } \\
\hline Median (min/max) & $27+1(24 / 33)$ & $26+5(23 / 28)$ \\
\hline \multicolumn{3}{|l|}{ Birth weight } \\
\hline Median, g (min/max) & $993(580-1,480)$ & $813(495-1,420)$ \\
\hline \multicolumn{3}{|l|}{ Removal of equipment } \\
\hline $\begin{array}{l}\text { Median, } n \text { (weeks), } \\
(\min / \max )\end{array}$ & $2.5(1-6)$ & $8.5(3-19)$ \\
\hline \multicolumn{3}{|c|}{ Colonization of CPAP equipment } \\
\hline Patients, $n(\%)$ & $1(12.5)$ & $11(55)$ \\
\hline $\begin{array}{l}\text { Time of detection, } \\
\text { weeks }\end{array}$ & 5 & 3.5 \\
\hline $\begin{array}{l}\text { Time of colonization, } \\
\text { weeks }\end{array}$ & 9 & $4.6(2-14)$ \\
\hline Microorganism $(n, \%)$ & S. aureus & $\begin{array}{c}\text { Enterobacteriacae }(6,55 \%) \\
\text { S. aureus }(2,18 \%) \\
\text { Fungi }(2,18 \%) \\
\text { P. aeruginosa }(1,9 \%)\end{array}$ \\
\hline
\end{tabular}

Colonization of Ambu bag

$\begin{array}{lcc}\text { Patients, } n & 0 & 3(17) \\ \text { Time of detection, } & 2.7 \\ \text { weeks } & \\ \text { Time of colonization, } & 4.3\end{array}$
weeks

Microorganism ( $n$, \%)

Enterobacteriacae $(2,50 \%)$ S. aureus $(1,25 \%)$

Colonization only in screening swabs

$$
\text { Patients, } n \text { (\%) }
$$

Time of detection,

$4(1-8)$ weeks

Time of colonization, weeks

Microorganism $(n, \%)$

$$
3(1-7)
$$

Enterobacteriacae (5, 63\%) Fungi $(2,25 \%)$ S. aureus $(1,13 \%)$

CPAP, continuous airway pressure; Max, maximum; Min, minimum.

During the study time eight infants developed infection/ sepsis with CoNS; six of them had positive blood cultures and in two of them CoNS was found on the tip of the central venous line. In all patients CoNS was found in swabs from the pharynx and in one case also on the CPAP equipment. No sepsis with Gram-negative bacteria occurred.

\section{DISCUSSION}

In this study we repeatedly performed a microbiological analysis at different sites of the CPAP equipment and found that the CPAP equipment in contact with the infant was colonized in up to $90 \%$ of cases. Over time, colonization with skin flora decreased and colonization with facultative 
Table 3. Facultative pathogenic microorganisms found in screening swabs from pharynx/anus

\begin{tabular}{cc}
\hline Patients $(n)$ & Type of microorganism \\
\hline Screening anal & \\
2 & Staphylococcus aureus \\
1 & Pseudomonas aeruginosa \\
4 & Escherichia coli \\
4 & Enterobacter cloacae \\
2 & Klebsiella oxytoca \\
1 & K. pneumoniae \\
1 & Morganella morganii \\
4 & Fungi \\
Screening pharynx & \\
3 & S. aureus \\
1 & Streptococcus agalactiae \\
3 & E. cloacae \\
4 & E. coli \\
3 & K. oxytoca \\
1 & K. pneumoniae \\
1 & P. aeruginosa \\
1 & Pantoea spp \\
1 & Fungis \\
2 &
\end{tabular}

pathogens (mainly Gram-negative bacteria) increased. CPAP colonization persisted over weeks in most infants. Ambu bags were used only for change of inspiratory limbs and were colonized less than CPAP equipment. If colonization of the CPAP equipment was present in patients with positive routine screening, it was identical with oropharyngeal/anal colonization, and both colonizations were detected at about the same time. No multidrug-resistant strains were identified during the period of surveillance, and respiratory infections were rare.

One-third of the infants had negative oropharynx and anal screening; two-thirds had positive screening, which was dominated by Enterobacteriacae. As expected, infants with positive oropharynx/anal screening tended to be younger, had a lower birth weight, a longer hospital stay, and needed the CPAP equipment for longer. These results are in line with others published: Smith et al. found that low birth weight was a risk factor for Gram-negative colonization (10). Milisavljevic et al. showed that Gram-negative bacteria increase in the gut with increasing hospital stay (11). Aly et al. found that nasal flora mainly consists of CoNS, but under CPAP Gramnegative bacteria can be detected in 38\% ( (ref. 4)). Possibly, Gram-negative selection of the infants' colonization is promoted by patient-side equipment such as CPAP.

In almost half of the infants, the CPAP equipment was colonized. The prongs and masks are in contact with secretions of the infant, and thus colonization with the isolates from the screening swabs is expected. In all but one patient colonization was the same as detected in routine screening. One patient hosted $S$. aureus on the equipment for 9 weeks, although this was never found in his pharyngeal or anal swabs. In seven patients oropharynx/anal screening was positive, but CPAP equipment never showed any colonization. The time of first detection and duration of colonization did not differ between infants with positive routine screening and CPAP equipment colonization vs. infants with positive routine screening without CPAP equipment colonization.

The reason why some infants had no colonization found on the equipment, whereas others had, was unclear. It is unlikely that over weeks microbes were not detected during the microbiological monitoring. If the CPAP equipment was colonized with facultative pathogenic microbes, Enterobacteriacae dominated. Brooks et al. found that gastric and respiratory tubes are a reservoir for gut microbes of premature infants (12). Meinich Petersen et al. reported that nasogastric feeding tubes yield high concentrations of facultative pathogenic bacteria even 1 day after insertion (7). Schwietz et al. reported that the interindividual gut flora of hospitalized preterm infants is more similar to that in breast-fed, full-term infants and is dominated by Gram-negative bacteria (13). Hewitt et al. showed that samples from different NICU surfaces were dominated by Enterobacteriacae, which commonly inhabit the digestive tract (14). Brooks et al. found that gut colonizers were widely distributed throughout the room environment (12). As we did not perform molecular typing, we cannot comment about the similarity of the detected isolates; moreover, our sample size was relatively small. We might have missed bacteria, because we used culture-based methods for detection. Thus, culture-independent methods based on amplification and sequencing of $16 \mathrm{~S}$ rRNA genes can detect more bacteria (14). However, the viability of these bacteria cannot be determined with these methods, and the question that bacteria cause infection cannot be answered.

Colonization of ambu bag masks and connectors was low because the equipment was used only for change of inspiratory limbs in infants dependant on CPAP. In infants who were able to make pauses on CPAP, ambu bags were not used anymore and were kept in the sterile cover, which was opened to be ready for use. Colonization of the ambu bags was identical with patients' own flora in all cases and was present only when the bags were in use.

Two of our patients on CPAP acquired pneumonia (2/26, $8 \%)$; our results in this small group of patients are in line with data published by others from much larger cohorts. The incidence of pneumonia in VLBW infants is $11-13 \%$ ( (refs $2,15)$ ). In both patients, E. cloacae was isolated from tracheal aspirates after respiratory deterioration. Cernada et al. published in 2013 that when using an invasive sampling technique of the lower respiratory tract such as a blindprotected catheter, non-contaminated samples from the lower respiratory tract could be obtained (16). Our infants had acquired pneumonia defined by clinical criteria (respiratory deterioration, more and purulent secretions, mild laboratory 


\section{Articles | Winking et al.}

findings, and no pathogen isolated in blood cultures) while on CPAP. The Gram-negative pathogen found also in the pharynx of both infants might be placed in the trachea during intubation and the causing pathogen might not have been found by us. Invasive sampling techniques have not been established in our unit and have not been a standard in neonatology so far. The low rate of pneumonia despite the high colonization rate should be interpreted with caution on how important or not colonization may be. It is possible that infants with colonized respiratory equipment are not at higher risk of pneumonia.

Neonatal infections among extremely low birth weight infants are associated with poor neurodevelopmental and growth outcomes (17). Therefore, it is a key question that which measures can reduce the incidence of infections and what role the equipment has in these infants. Hand hygiene of course has the most important role. Selective digestive tract decontamination or selective oropharyngeal decontamination has not significantly reduced ventilator-associated pneumonia $(18,19)$, but has led to antibiotic-resistant intestinal flora $(20)$. Probiotics reduce the risk of necrotizing enterocolitis and mortality, but not of sepsis or pneumonia (21). Restrictive use of antibiotics leads to a lower rate of colonization with Gramnegative bacteria $(22,23)$.

Our data show that colonization of the infants and their respiratory equipment starts early, persists over weeks, and is similar to their gastrointestinal flora. Preterm infants acquire microorganisms from their NICU environment and the direction of colonization of the equipment-whether from the environment primarily to gastric and respiratory tubing or from the gastric tract to the equipment-is not clear yet. Frequent cleaning of the CPAP equipment with $\mathrm{NaCl} 0.9 \%$ had not prevented respiratory infections in this small group of infants. Whether shorter changing intervals or more disinfection of the CPAP equipment will lower rates of respiratory infections should be considered and investigated in further studies.

Disclosure: The authors declare no conflict of interest.

\section{REFERENCES}

1. Adams-Chapman I, Stoll BJ. Prevention of nosocomial infections in the neonatal intensive care unit. Curr Opin Pediatr 2002;14:157-64.

2. Geffers C, Baerwolff S, Schwab F, Gastmeier P. Incidence of healthcareassociated infections in high-risk neonates: results from the German surveillance system for very-low-birthweight infants. J Hosp Infect 2008;68:214-21.

3. Cernada M, Brugada M, Golombek S, Vento M. Ventilator-associated pneumonia in neonatal patients: an update. Neonatology 2014;105: 98-107.

4. Aly H, Hammad TA, Ozen M, et al. Nasal colonization among premature infants treated with nasal continuous positive airway pressure. Am J Perinatol 2011;28:315-20.

5. Graham PL III, Begg MD, Larson E, Della-Latta P, Allen A, Saiman L. Risk factors for late onset gramnegative sepsis in low birth weight infants hospitalized in the neonatal intensive care unit. Pediatr Infect Dis 2006;25:113-7.

6. Gupta AK, Anand NK, Manmohan NK, Lamba IMS, Gupta R, Srivastava L. Role of bacteriological monitoring of the hospital environment and medical equipment in a neonatal intensive care unit. J Hosp Infect 1991;19: 263-71.

7. Meinich Petersen S, Greisen G, Krogfelt KA. Nasogastic feeding tubes from a neonatal department yield high concentrations of potentially pathogenic bacteria - even $1 \mathrm{~d}$ after insertion. Pediatr Res 2016;80:395-400.

8. Pneumatikos IA, Dragoumanis CK, Bouros DE. Ventilator-associated pneumonia or endotracheal tube- associated pneumonia?: An approach to the pathogenesis and preventive strategies emphasizing the importance of endotracheal tube. J Am Soc Anesthesiol 2009;110:673-80.

9. Robert Koch-Institut. Commission for hospital hygiene and infection prevention Microbiological screening of colonization of preterm and newborn infants in ICUs. Epid Bull 2013;42:421-32.

10. Smith A, Saiman L, Zhou J, Della-Latta P, Jia H, Graham PL. III. Concordance of gastrointestinal tract colonization and subsequent bloodstream infections with gramnegative bacilli in very low birthweight infants in the neonatal intensive care unit. Pediatr Infect Dis J 2010;29:831.

11. Milisavljevic V, Garg M, Vuletic I, et al. Prospective assessment of the gastroesophageal microbiome in VLBW neonates. BMC Pediatr 2013;13:49.

12. Brooks B, Firek A, Miller CS, et al. Microbes in the neonatal intensive care unit resemble those found in the gut of premature infants. Microbiome 2014;2:1.

13. Schwiertz A, Gruhl B, Löbnitz M, Michel P, Radke M, Blaut M. Development of the intestinal bacterial composition in hospitalized preterm infants in comparison with breast-fed, full-term infants. Pediatr Res 2003;54:393-9.

14. Hewitt KM, Mannino FL, Gonzalez A, et al. Bacterial diversity in two neonatal intensive care units (NICUs). PLoS ONE 2013;8:e54703.

15. Couto RC, Pedrosa TM, Tofani CP, Pedroso ER. Risk factors for nosocomial infection in a neonatal intensive care unit. Infect Control Hosp Epidemiol 2006;27:571-5.

16. Cernada M, Aguar M, Brugada M, et al. Ventilator-associated pneumonia in newborn infants diagnosed with an invasive bronchoalveolar lavage technique: a prospective observational study. Pediatr Crit Care Med 2013;14:55-61.

17. Stoll BJ, Hansen NI, Adams-Chapman I, et al. National Institute of Child Health and Human Development Neonatal Research Network (2004). Neurodevelopmental and growth impairment among extremely lowbirth-weight infants with neonatal infection. JAMA 2004;292:2357-65.

18. Keyt H, Faverio P, Restrepo MI. Prevention of ventilator-associated pneumonia in the intensive care unit: a review of the clinically relevant recent advancements. Indian J Med Res 2014; 139:814.

19. Philips BJ. Selective decontamination of the digestive tract: time to implement it in all UK intensive care units? Maybe not yet. Br J Anaesth 2014;13:537-9.

20. Strenger V, Gschliesser T, Grisold A, et al. Orally administered colistin leads to colistin-resistant intestinal flora and fails to prevent faecal colonization with extended-spectrum beta-lactamase-producing enterobacteria in hospitalized newborns. Int J Antimicrob Agents 2011;37:67-9.

21. Olsen R, Greisen G, Schroder M, Brok J. Prophylactic probiotics for preterm infants: a systematic review and meta-analysis of observational studies. Neonatol 2016;109:105-2.

22. Calil R, Marba ST, von Nowakonski A, Tresoldi AT. Reduction in colonization and nosocomial infection by multiresistant bacteria in a neonatal unit after institution of educational measures and restriction in the use of cephalosporins. Am J Infect Control 2001;29:133-8.

23. Jacquot A, Neveu D, Aujoulat F, et al. Dynamics and clinical evolution of bacterial gut microflora in extremely premature patients. J Pediatr 2011;158:390-6. 